\title{
Enhancing the network synchronizability
}

\begin{abstract}
The structural and dynamical properties, particularly the small-world effect and scale-free feature, of complex networks have attracted tremendous interest and attention in recent years. This article offers a brief review of one focal issue concerning the structural and dynamical behaviors of complex network synchronization. In the presentation, the notions of synchronization of dynamical systems on networks, stability of dynamical networks, and relationships between network structure and synchronizability, will be first introduced. Then, various technical methods for enhancing the network synchronizability will be discussed, which are roughly divided into two classes: Structural Modification and Coupling-Pattern Regulation, where the former includes three typical methods--dividing hub nodes, shortening average distances, and deleting overload edges, while the latter mainly is a method of strengthening the hub-nodes' influence on the network.
\end{abstract}

Keywords complex networks, synchronization, structural modification, coupling pattern

\section{PACS numbers 89.75.-k, 05.45.Xt}

ZHAO Ming, CHEN Guan-rong $(\bowtie)$

Department of Electronic Engineering, City University of Hong Kong, Kowloon, Hong Kong SAR, China

E-mail: eegchen@cityu.edu.hk

ZHAO Ming, ZHOU Tao, WANG Bing-hong

Department of Modern Physics and Nonlinear Science Center, University of Science and Technology of China, Hefei 230026, China

\section{ZHOU Tao}

Department of Physics, University of Fribourg, Chemin du Muse 3, CH1700 Fribourg, Switzerland

WANG Bing-hong

Institute of Complex Adaptive Systems, Shanghai Academy of System Science, Shanghai 200093, China

\section{Introduction}

To study the collective behaviors of complex systems, a common way is to represent each individual system by a node and the interaction between two individuals by an edge, thereby abstracting the coupled systems as a network. Then, the study is correspondingly converted to investigating the dynamical behaviors of the resultant network. Within the context of general networks, random networks have been the most well-studied ones in the recent half-century. In recent years, the speedy development of computer science and the rapid evolvement of the Internet have provided researchers with super-computing power and huge databases for much more efficient network studies, leading to the discoveries of small-world effect and scale-free property of networks, and further to the construction of some new network models which are more realistic and in many aspects closer to real-world networks in social, biological and technological systems. These new models, particularly the small-world and scale-free network models [1,2], characterize various complex networks by such measures as average distance, clustering coefficient, power-law degree distribution, and so on [3-6].

A particular subject in network research is concerned with the synchronization of coupled dynamical systems (as nodes) on a complex network, and this important subject has been extensively investigated in the past few years [7-15]. In retrospect, the investigation of synchronization of dynamical systems on complex networks has gone through several stages. At the very beginning, scientists eagerly studied the synchronizability of two typical kinds of network models and found that coupled dynamical oscillators on small-world and scale-free network models are much easier to synchronize than on regular lattices, and the synchronizability of a small-world network model is even better than that of random networks in some cases [16-19]. It was then intuitively believed, that the 
strong synchronizability of such networks is due to their short average distances, however, it was lately found [20] that in some complex networks, with the increasing of heterogeneity of degree and betweenness distributions, although the average distance becomes shorter, the network synchronizability actually becomes worse. The subsequent works on possible relationships between network structures and the synchronizability have shown that a combination of shorter average distance and more homogeneous degree distribution can make the network to achieve synchronization easier [20-24]. Thereafter, great efforts have been devoted to seeking ways to enhance the network synchronizability, and this remains one of the most active research activities in the field today.

This article offers a brief review of the focal issue concerning the enhancement of network synchronizability. The basic notions of synchronization of dynamical systems on networks, stability of dynamical networks, and relationships between network structure and synchronizability, will be first introduced. Then, some technical methods for enhancing the network synchronizability will be discussed; they are divided into two classes: Structural Modification, which includes three typical methods-dividing hub nodes, shortening average distances, and deleting overload edges, and Coupling-Pattern Regulation, which is mainly a method of strengthening the hub-nodes' influence on the network. Finally in this article, some open questions will be posed, which are deemed interesting and significant thus might further enlighten the readers' interests in this attractive research subject.

\section{Network models and the master stability function}

In this section, a generic model of coupled dynamical systems on a network is considered, and the master stability function approach is taken to study the stability of the fully synchronized states of the network [25-28].

In the network, each node is located with a dynamical system such as an oscillator, and an edge connecting two nodes means that there exits a bi-directional coupling between them. Denote the state of the $i$ th node by $\boldsymbol{x}_{i}, i=1, \cdots, N$, and the set of dynamical equations for the whole network are obtained as

$$
\dot{\boldsymbol{x}}_{i}=\boldsymbol{F}\left(\boldsymbol{x}_{i}\right)-\sigma \sum_{j=1}^{N} G_{i j} \boldsymbol{H}\left(\boldsymbol{x}_{j}\right), \quad i=1, \cdots, N
$$

where $\dot{\boldsymbol{x}}_{i}=\boldsymbol{F}\left(\boldsymbol{x}_{i}\right)$ describes the dynamics of each individual node, $\sigma$ is the coupling strength, and $\boldsymbol{H}\left(\boldsymbol{x}_{j}\right)$ is the output function. In this model, $G_{i j}$ are the elements of the coupling matrix $G, i, j=1, \cdots, N$, which is determined by the cou- pling pattern among the nodes; for example, when the nodes are coupled symmetrically, $G$ has the same form as the network Laplacian $L$, i.e., $G=L$, where

$$
L_{i j}=\left\{\begin{array}{cl}
k_{i}, & \text { for } i=j \\
-1, & \text { for } j \in \Lambda_{i} \\
0, & \text { otherwise }
\end{array}\right.
$$

in which $k_{i}$ is the degree of node $i$ and $\Lambda_{i}$ is the set of node $i$ 's neighbors.

Because of the irreducible and diffusive coupling structure and the symmetry and positive semi-definiteness of $L$, it has zero row sums and all eigenvalues are nonnegative real and the smallest eigenvalue (denoted by $\lambda_{1}$ ) is always zero with multiplicity 1 . Thus, the eigenvalues can be ranked as $0=$ $\lambda_{1}<\lambda_{2} \leqslant \lambda_{3} \leqslant \cdots \leqslant \lambda_{N}$. In this setting of the network, the synchronization manifold is invariant, on which the fully synchronized state satisfies $\boldsymbol{x}_{1}=\boldsymbol{x}_{2}=\cdots=\boldsymbol{x}_{N}=\boldsymbol{s}$, with $\dot{s}=\boldsymbol{F}(s)$.

For some dynamical systems, the master stability function is negative when all the products of the coupling strength $\sigma$ and the nonzero eigenvalues of the Laplacian $L$ are larger than a certain constant $\alpha_{1}>0$, so that the largest Lyapunov exponent of the connected network is negative [25, 26]. Therefore, the network is synchronizable when the eigenvalue $\lambda_{2}>\alpha_{1} / \sigma$. In this case, the larger the $\lambda_{2}$, the better the network synchronizability. For some other dynamical systems, however, the master stability function is negative only in a finite interval $\left(\alpha_{1}, \alpha_{2}\right)$ [29], over which the largest Lyapunov exponent of the network is negative, so that the network is synchronizable for some $\sigma$ when the eigenratio $R=\lambda_{N} / \lambda_{2}$ satisfies $R<\alpha_{2} / \alpha_{1}[27,28]$. In this case, the smaller the $R$, the better the network synchronizability. The former corresponds to the case that the stability zone is unbounded, and the latter, bounded. As a matter of fact, these two cases can also co-exist [30-33]. Notice, moreover, that the right-hand of the two inequalities depend only on the dynamics of the individual system and the output function, while the eigenvalue $\lambda_{2}$ and the eigenratio $R$ depend only on the Laplacian $L$. Consequently, the problem of synchronization can be divided into two parts: choosing suitable parameters of node dynamics and analyzing the eigenvalues of the Laplacian. The stability analysis is also valid for some more complicated coupling patterns [25-28, 34], including the case when $G$ is nondiagonalizable [35].

\section{Network structures and the synchronizability}

As mentioned above, when the average distance keeps small, 
the more heterogenous the degree or the betweenness distribution, the more difficult the networks achieves synchronization. Nishikawa et al. [20] gave an explanation of this phenomenon: when the nodes are coupled symmetrically, the hubs (nodes of large degrees) receive too many signals (or too much information) at the same time, so the signals may cancel one another, resulting in effectively no communications passing through the hubs. They also pointed out that edges with larger loads may encounter the same situation. Later, in comparison to the results of [20], Hong et al. [21] concluded that the node with the maximal betweenness is the bottleneck for network synchronization, so it can be used as an indicator for the network synchronizability: the smaller the maximal betweenness, the better the network synchronizability. Although later it is found that the maximal betweenness is not a suitable indicator for network synchronizability [23, 33, 36], this claim may still be valid to some extent.

Based on the knowledge about the relations between the network structure and its synchronizability, it is easy to conclude that to improve the network synchronizability, the heterogeneity of degree or betweenness distribution, or the average distance, or the maximal betweenness (load) of a network should be reduced. This ideas leads to the classification of two types of methods for enhancing the network synchronizability: one is to modify the network structure and the other is to regulate the coupling pattern. In the subsequent section, four specific methods of these two types will be discussed respectively.

\section{Some methods for enhancing network synchroniza- bility}

In this section, two specific methods are introduced, which can enhance the network synchronizability effectively: modifying the network structure and regulating the coupling pattern.

\subsection{Dividing hub nodes}

In Ref. [21], Hong et al. calculated the eigenratio of the Laplacian before and after the removal of the node with the maximal betweenness. It turns out that after the removal, the eigenratio is sharply reduced, indicating an improvement of the network synchronizability. It is an effective way to test the role of the hub-node, but is not practical because the hubnode in a real-world network is very important and cannot be removed freely. Nevertheless, this may be got around by, for instance, letting other nodes to share the load of a hub.
Inspired by the aforementioned idea, a method named structural perturbations was developed in Ref. [37] to improve the network synchronizability. Specifically, for a hub $x_{0}, m-1$ auxiliary nodes, labelled as $x_{1}, x_{2}, \cdots, x_{m-1}$, are added around it and let them be fully connected. Then, each edge of $x_{0}$ will be relinked to a randomly picked node $x_{i}$, $i=0,1, \cdots, m-1$. After this process, the betweenness of $x_{0}$ is divided into $m$ almost equal parts associating with these $m$ nodes. As a result, the load on the hub $x_{0}$ has been almost uniformly redistributed among the $m$ nodes. The process is called $m$-division for short. A sketch map of a 3-division process on node $x_{0}$ is shown in Fig. 1.

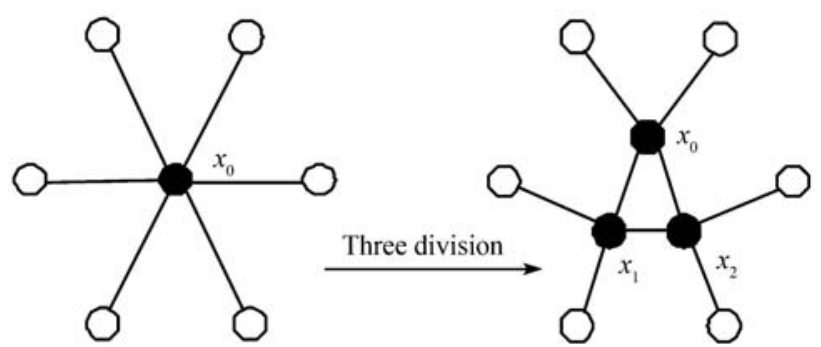

Fig. 1 Sketch maps for the 3-division process on $x_{0}$. The solid circle on the left is the node $x_{0}$ with degree 6 . After the 3 -division process, $x_{0}$ is divided into 3 nodes, $x_{0}, x_{1}$ and $x_{2}$, that are fully connected each other. The six-edge incident from $x_{0}$ redistribute over these three nodes (after Ref. [37]).

Due to the huge size of many real-life networks, it is usually impossible to obtain the nodes' betweenness. Fortunately, previous studies show that there exists strongly positive correlation between degree and betweenness in BarabásiAlbert (BA) networks and some other real heterogeneous networks $[38,39]$; that is, the nodes with larger degrees will statistically have larger betweenness. Therefore, for practical reason, it can be assumed that a node with higher betweenness also has a larger degree in BA networks.

In order to enhance the network synchronizability, a few nodes with the highest degrees will be divided by the abovedescribed $m$-division process. Rank all nodes of a given network $G$ according to their degrees in decreasing order, so the node has the highest degree is arranged in the top of the queue. Then, a new network $G(\rho, m)$ can be obtained in the following $N \rho$ steps. Firstly, carry out $m$-division on the top node in $G$, yielding a new network $G\left(\frac{1}{N}, m\right)$. Secondly, calculate all nodes' degrees in $G\left(\frac{1}{N}, m\right)$, and rank them again in the same way. Thirdly, generate network $G\left(\frac{2}{N}, m\right)$ by dividing the top node in $G\left(\frac{1}{N}, m\right)$ in the same way. Finally, repeat this process $N \rho$ times, during which totally $N \rho$ nodes will be 
divided, thus network $G(\rho, m)$ is obtained. Since the edges are relinked to the $m$ nodes at random in the dividing process, $G(\rho, m)$ is generally not unique, but this is not important.

To explore how the structural perturbations affect the network synchronizability, the eigenratios $r$ before and after the dividing processes are now compared. A BA network of size $N=2000$ with average degree $\langle k\rangle=12$ is used for simulation. In Fig. 2, the ratio $R=r^{\prime} / r$ is plotted against the number of nodes that are divided, where $r$ is the eigenratio of the original network and $r^{\prime}$, the one after the process. Here, $m=3$. With the probability $\rho$ or the number of divided nodes increasing, the ratio $R$ is decreasing, indicating the enhancement of the synchronizability. In Fig.2, it can be seen that dividing only a few nodes is already able to enhance the network synchronizability prominently. For example, when $R$ decreases to 0.7 while only 5 nodes are divided, the ratio will drop to half after only $0.6 \%$ nodes (i.e., 12 nodes) are divided.



Fig. 2 Behavior of the ratio $R$, between the eigenratio of network after node-division and that of the network before the division, versus the fraction of the divided nodes $\rho$. As the number of the divided nodes increases, the ratio $R$ is reduced, leading to better synchronization (after Ref. [37]).

To better understand the underlying mechanism of synchronization and the reason why these structural perturbations can significantly enhance the network synchronizability, the behaviors of two extensively studied quantities, the average distance $L$ and the maximal degree $k_{\max }$, are further examined. In a BA network, the node with the maximal degree is most likely the only node having the maximal betweenness. As illustrated by Fig. 3, $L$ will increase with $\rho$, while $k_{\max }$ will decrease. This results provide some evidences for the way the two factors affect the synchronization of the network. The maximal degree (and, the maximal betweenness) may play a major role in determining the network synchronizability. It is worthwhile to emphasize that from the simulation results, one cannot say anything about how the aver- age distance affects the network synchronizability. When $L$ is varied slightly, the probably has insignificant influence on the synchronization as compared with the change of $k_{\max }$. This suggests that reducing the maximal betweenness of a network is a practical and effective approach to enhancing the network synchronizability.

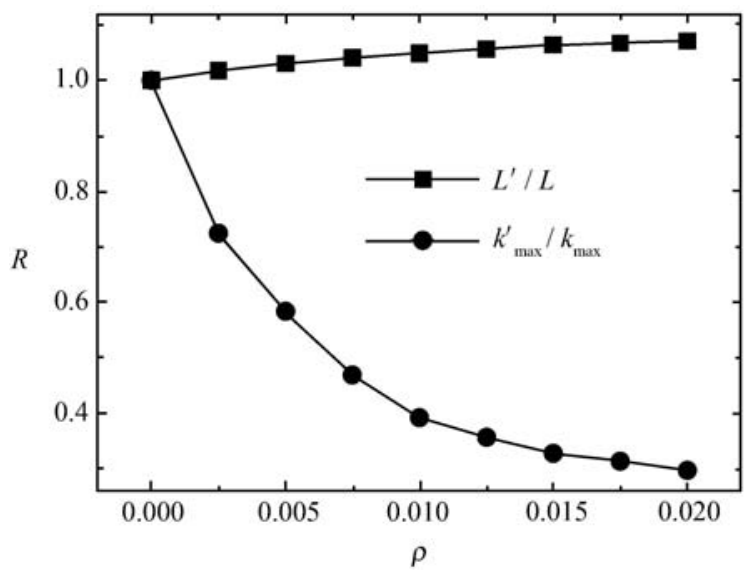

Fig. 3 The average distance $L^{\prime}$ and maximal degree $k_{\max }^{\prime}$ in $G(\rho, m)$ versus $\rho$. Here, $L$ and $k_{\max }$ denote the average distance and maximal degree in the original network $G$. The relative changes $L^{\prime} / L$ and $k_{\max }^{\prime} / k_{\max }$ are plotted using squares and circles, respectively. It is clear that the dividing processes reduce the maximal degree while increase the average distance (after Ref. [37]).

\subsection{Shortening the average distance}

In a network, if the degree distribution keeps fixed, shortening the average distance will enhance the network synchronizability. Based on this observation, Zhou et al. [40] investigated the synchronizability of a network model named crossed double cycles (CDCs), not only testifying the relationship between the average distance and the network synchronizability but also providing a possible way to make network more synchronizable.

In the language of graph theory [41-43], the cycle $C_{N}$ denotes a network consisting of $N$ vertices, $x_{1}, x_{2}, \cdots, x_{N}$. These $N$ vertices are arranged as a ring, where the nearest two vertices are connected together. Hence, $C_{N}$ has $N$ edges connecting the vertices $x_{1} x_{2}, x_{2} x_{3}, \cdots, x_{N-1} x_{N}, x_{N} x_{1}$. The CDCs, denoted by $G(N, m)$, can be constructed by adding two edges, called crossed edges, to each vertex in $C_{N}$. The two vertices connecting by a crossed edge have distance $m$ in $C_{N}$. For example, network $G(N, 3)$ can be constructed from $C_{N}$ by connecting $x_{1} x_{4}, x_{2} x_{5}, \cdots, x_{N-1} x_{2}, x_{N} x_{3}$. And network $G(N, 2)$ is isomorphic to a one-dimensional lattice with periodic boundary conditions and with coordination number 
$z=2$ [44]. A network $G$ is isomorphic to a network $H$ if there exists a bijective map $\theta: V(G) \rightarrow V(H)$, satisfying the adjacency-preserving condition $(x y) \in E(G) \Longleftrightarrow$ $(\theta(x) \theta(y)) \in E(H)$, where $V(\cdot)$ and $E(\cdot)$ denote the sets of vertices and edges, respectively. Roughly speaking, two networks are isomorphic if they have the same structure.

A sketch map of $G(20,4)$ is shown in Fig. 4. Clearly, all the vertices in $G(N, m)$ are of degree 4, thus the variance of degree is equal to 0 . Furthermore, $G(N, m)$ is vertextransitivity; that is, for any two vertices $x$ and $y$ in $G(N, m)$, there exists an automorphism map $\theta: V(G) \rightarrow V(G)$ such that $y=\theta(x)$. The vertex-transitivity networks are completely symmetrical, which are of particular practicability in the design of topological structures of data memory allocation and multiple processor systems [45].



Fig. 4 (Color online) Sketch maps of $G(20,4)$ (after Ref. [40]).

Figure 5 shows how the average distance $L$ affects the network synchronizability (again, measured by the eigenratio $R$ ) when the nodes are coupled symmetrically. One can see clearly that the network synchronizability is very sensitive to the average distance; as $L$ increases, the eigenratio $R$ sharply spans more than three magnitudes. And the network synchronizability is remarkably enhanced by simply reducing $L$. When the crossed length $m$ is not too small or too large (compared with $N$ ), the networks with the same average distance have approximately the same synchronizability, no matter what these network sizes are. More interestingly, the numerical results indicate that the eigenratio $R$ approximately obeys a power-law form as $R \sim L^{1.5}$ (see the inset of Fig. 5).

\subsection{Deleting overload edges}

In the synchronization process, not only the hub-nodes may

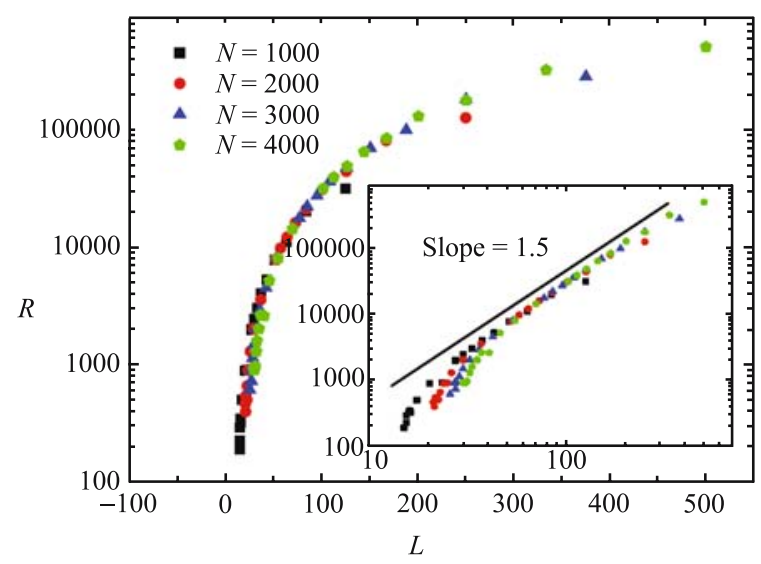

Fig. 5 (Color online) The eigenratio $R$ versus the average distance $L$ in CDCs. The black squares, red circles, blue triangles and green pentagons represent the cases of $N=1000,2000,3000$ and 4000, respectively. The inset shows the same data in a log-log plot, indicating that the eigenratio $R$ approximately obeys a power-law form as $R \sim L^{1.5}$. The solid line has slope 1.5, just for comparison (after Ref. [40]).

be the bottlenecks but also some edges with larger loads may trouble the network synchronizability. Yin et al. [46] found that in scale-free networks, when the nodes are coupled symmetrically, if some overload edges (i.e., the edges having largest loads) are removed, the network will become more synchronizable. To get the load of each edge need the global information of the network and is of huge computational complexity. To avoid the heavy computational cost, they define the significance of an edge $S_{i j}$ by the product of the degrees of two nodes $i$ and $j$ at both sides of the edge, i.e., $S_{i j}=k_{i} \times k_{j}$. After calculating the significance of all the edges, they rank the edges according to the values $S_{i j}$. Subsequently, at each time step, an edge with the highest rank is cut, i.e., decouple the tow nodes at both sides of the edge.

Figure 6 shows the ratio of the eigenratios of the coupling matrix after and before the edges are deleted, as a function of the proportion of the cut-edges. It is clear that as the number of deleted edges increases, the synchronizability is improved, reflected by the decrease of the eigenratio. But the average degree seems has little influence on the results.

\subsection{Strengthening the hub-nodes' influence}

From the discussion in Section 2, it is known that when the nodes are coupled symmetrically, it is usually hard for the coupling signals to pass through the hub-nodes. Thus, during the coupling process, the states of the hub-nodes are influenced insignificantly, and the nodes around the hub-nodes will approach the states of the hub-nodes, little by little, but eventually all the nodes are synchronized. Therefore, 


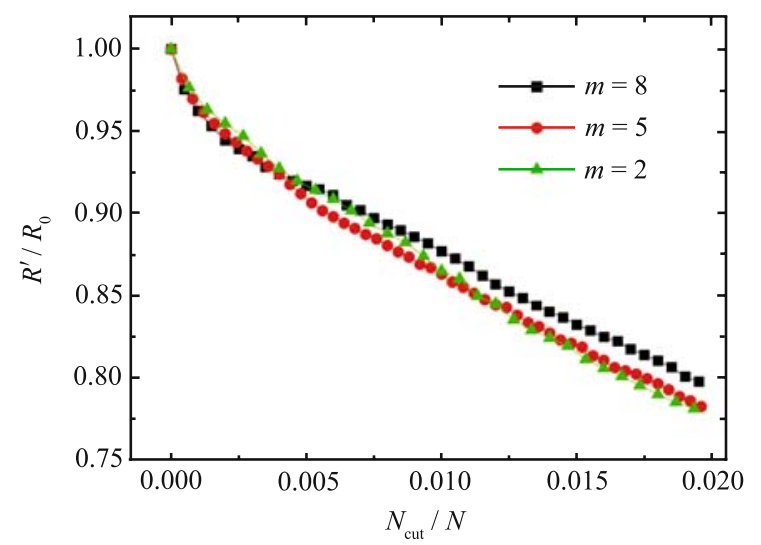

Fig. 6 (Color online) Changes of the synchronizability as a function of the proportion of the cut-edges $N_{\text {cut }} / N$, for different values of the average distance (after Ref. [46]).

if the coupling strength from hub-nodes to other nodes are stronger than the reverse, i.e., the nodes are coupled asymmetrically, then the network may actually synchronize much easier. Based on this obseervation, Motter et al. [47-49] proposed a new coupling pattern, in which the coupling matrix has zero row-sums, and the off-diagonal elements are $G_{i j}=L_{i j} / k_{i}^{\beta}$, where $\beta$ is a tunable parameter and $L_{i j}$ are the elements of the Laplacian, $i, j=1, \cdots, N$. The coupling is weighted when $\beta \neq 0$, but unweighted when $\beta=0$. It was proved that network nodes coupled in this way are much easier to synchronize than being coupled symmetrically, and $\beta=1$ is always the best case.

The aforementioned coupling method only takes into account the degree difference between the nodes that are directly connected, where each node is coupled by its neighbors with the same coupling strength. However, in some realworld networks, different neighbors may have different influences, i.e., the coupling strengths from different neighbors may be different. Thus, based on the assumption that different nodes play different roles in a network, one may adjust the influence strength of each node, received from their neighbors, according to its neighbors' degrees [50]. The coupling matrix so designed is as follows:

$$
G_{i j}=\left\{\begin{array}{cc}
-k_{j}^{\alpha} / S_{i}^{\beta}, & \text { for } j \in \Lambda_{i} \\
S_{i} / S_{i}^{\beta}, & \text { for } i=j \\
0, & \text { otherwise }
\end{array}\right.
$$

where $S_{i}=\sum_{j \in \Lambda_{i}} k_{j}^{\alpha}, i=1, \cdots, N$. When $\alpha=\beta=0$, this coupling pattern degenerates to the symmetric coupling pattern [27], where the case of $\alpha=0$ corresponds to the MZK pattern [47], and the case of $\beta=1$ is equivalent to the one introduced in Ref. [49] [see Eq. (15) in Ref. [49] for more details].

Although $G$ is asymmetric for all $\alpha$ with $\beta \neq 0$, it can be proved that all its eigenvalues are non-negative real with only one eigenvalue being equal to zero if the network is connected. To do so, first notice that, Eq. (3) can be written as

$$
G=D L^{\prime}
$$

where $D=\operatorname{diag}\left\{k_{1}^{-\alpha} S_{1}^{-\beta}, k_{2}^{-\alpha} S_{2}^{-\beta}, \cdots, k_{N}^{-\alpha} S_{N}^{-\beta}\right\}$ is a diagonal matrix, and $L^{\prime}=\left(L_{i j}^{\prime}\right)$ is a symmetric matrix with zero row-sums, whose off-diagonal elements are $L_{i j}^{\prime}=$ $k_{i}^{\alpha} k_{j}^{\alpha}, i, j=1, \cdots, N$. From the identity

$$
\operatorname{det}\left(D L^{\prime}-\lambda I\right)=\operatorname{det}\left(D^{\frac{1}{2}} L^{\prime} D^{\frac{1}{2}}-\lambda I\right)
$$

which is valid for arbitrary $\lambda$, where "det" denotes the determinant and $I$ is the $N \times N$ identity matrix, one can deduce that the spectrum of eigenvalues of matrix $G$ is equal to the spectrum of a symmetric matrix defined by

$$
H=D^{\frac{1}{2}} L^{\prime} D^{\frac{1}{2}}
$$

As a result, the eigenvalues of matrix $G$ are all nonnegative real and the smallest eigenvalue is always zero.

In this coupling method, for a given parameter $\beta$, and for any $\alpha>0$, the nodes with larger degrees have stronger influence, but for $\alpha<0$, the nodes with less edges are more influential. Parameter $\beta$ is now exploited so as to eliminate the discrepancies between the coupling signals that each node receives: Given $\alpha$, when $\beta=1$, each node receives the equal quantum of signals; when $\beta<1$, the nodes having a larger sum of neighbors' degrees are being influenced more strongly; when $\beta>1$, the contrary is true.

Figure 7(a) shows some numerical values of the eigenratio $R$ on the parameter space $(\alpha, \beta)$ for BA networks [2]. To clearly show the effects of $\alpha$ and $\beta$ on $R$, the values of $R$ are plotted as a function of $\alpha$ for different values of $\beta$ in Fig. 7(b). No matter what value the parameter $\beta$ takes, there exists a region of $\alpha$ in which the eigenratio $R$ is smaller than that of the case $\alpha=0$. That is, when proper parameters are chosen, this coupling method can be even better than the MZK method. Similarly to the results obtained by the MZK method, $\beta=1.0$ corresponds to the case of best synchronizability. Hereinafter, the discussion will be focused on the case of $\beta=1$.

Note that, in the limit $\alpha=+\infty(-\infty)$, each node is only influenced by a neighbor having the largest (smallest) degree. Similar situation as mentioned in Ref. [51] appears here: The original network approaches a new configuration connected by some effective directed edges. And the new network, named an equivalent network, may be connected or disconnected. In the disconnected case, the eigenratio $R$ will approach infinity, while in the connected case, the eigenratio 


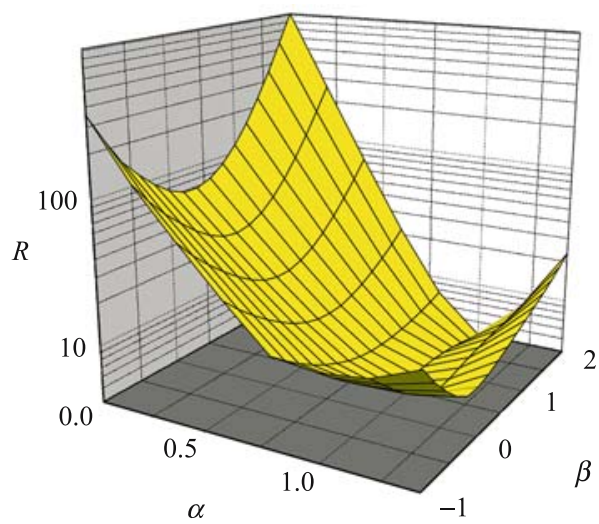

(a)

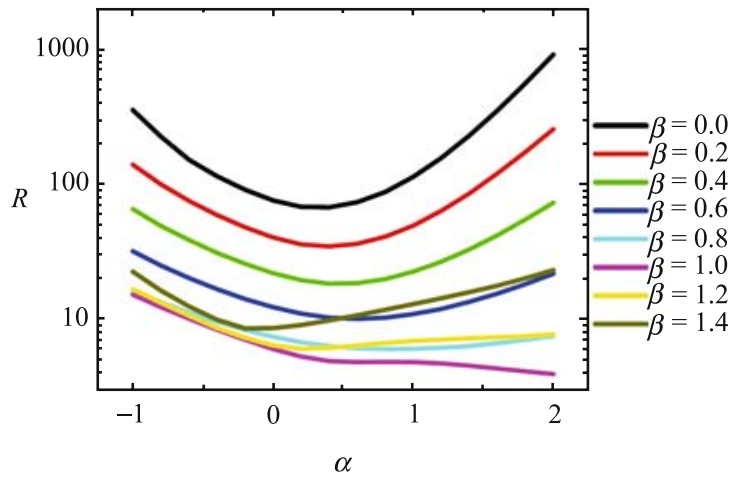

(b)

Fig. 7 (color online) (a) $R$ in the parameter plane $(\alpha, \beta)$; (b) $R$ versus $\alpha$ for different values of the parameter $\beta$. The numerical simulations are implemented based on a BA network of size $N=1024$ with average degree $\bar{k}=6$ (after Ref. [50]).

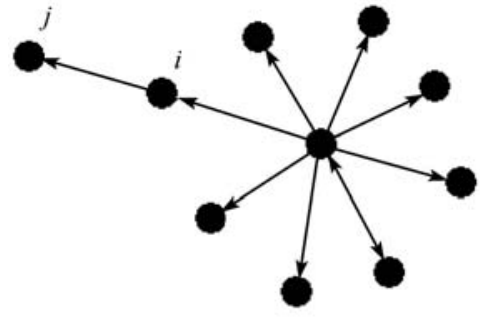

(a)

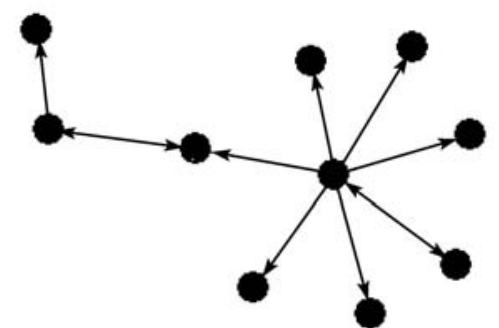

(b)

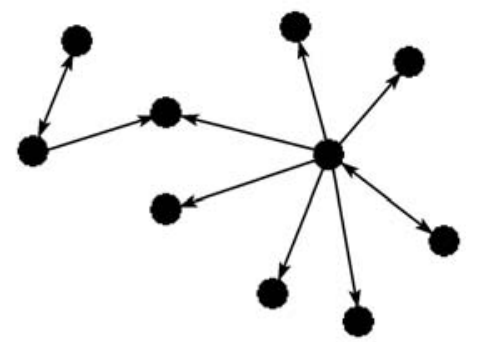

(c)

Fig. 8 Sketch maps of three simple equivalent networks, where the arrow from node $i$ to node $j$ indicates that the latter receives coupling signal from the former. Their eigenratios are 2 (a), 6.8284 (b) and $+\infty$ (c), respectively (after Ref. [50]).

equals a constant larger than or equal to 2 . Figure 8 illuminates three simple equivalent networks with $\alpha=+\infty$; the former two are connected, and the third one is disconnected. Their eigenratios are 2, 6.8284 and $+\infty$, respectively. Figure 9 shows the changes of the eigenratio $R$ with the parameter $\alpha$, when $\beta=1$, on different network configurations. When $\alpha>0.4$, the eigenratios for different configurations fall apart: some approach 2 or constants not much larger than 2, while some go to infinity, depending on whether the equivalent networks are connected or not. In addition, from the simulations, one can see that with the increasing of the network size, the proportion of networks being disconnected, when $\alpha=\infty$ $(-\infty)$, will increase sharply.

The effect of degree-degree correlation on the network synchronizability has also been investigated [52]. Some correlated networks are generated by an extended BA algorithm [53, 54]: starting from $m_{0}$ fully connected nodes; then, at each time step, a new node is being added to the network and $m\left(<m_{0}\right)$ previously existing nodes are chosen to be connected to it, with probability

$$
p_{i} \propto \frac{k_{i}+k_{0}}{\sum_{j}\left(k_{j}+k_{0}\right)}
$$

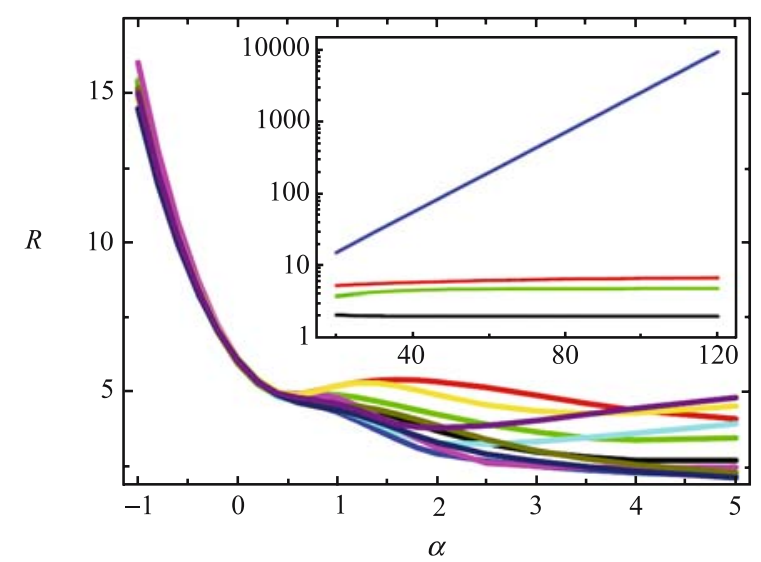

Fig. 9 (color online) The eigenratio $R$ versus the parameter $\alpha$ when $\beta=$ 1.0, for several BA network configurations of size $N=1024$ with average degree $\bar{k}=6$. Each color represents one configuration (after Ref. [50]).

where $p_{i}$ and $k_{i}$ denote the choosing probability and degree of node $i$, respectively, $i=1,2, \cdots$. By varying the free parameter $k_{0}(>-m)$, one can obtain the desired scale-free networks with different assortative coefficients $r[55,56]$.

Figure 10 shows the relationship between the eigenratio $R$ and the parameter $\alpha$, for different assortative coefficients, when $\beta=1$. Interestingly, there exists a unique cross point at 
$\alpha_{\text {cro }} \approx-0.25$. When $\alpha<\alpha_{\text {cro }}$, a stronger assortative value implies a better synchronizability, while when $\alpha>\alpha_{\text {cro }}$, the opposite is true. It can be proved that in networks of different sizes, the unique cross point always exits, but its position may change with the network size.



Fig. 10 (color online) The eigenratio $R$ versus the parameter $\alpha$, when $\beta=$ 1.0 , for the generalized BA networks with different assortative coefficients $r$ (after Ref. [50]).

\section{Conclusion and discussion}

This brief review has introduced some recent works on the synchronization of complex networks, especially some methods aiming at improving the network synchronizability. These methods can be divided into two classes, one is to modify the network structure and the other is to regulate the coupling pattern. In the former case, the network structure is changed according to some given rules; in the latter case, the network structure is kept unchanged while the coupling pattern is regulated to strengthen the influence of the hub-nodes on the entire network.

The study on network synchronizability is a rapidly growing area of research, attracting many scientists of various fields, from physics to engineering, to mathematics, to biology alike. Despite the encouraging and promising recent progress, there are still many open questions for further study. For instance, in the case of static coupling patterns, Nishikawa and Motter [35] pointed out that the optimal global synchornizability, with eigenratio being equal to 1 , can be obtained from a directed structure without any loops. Even if adding one loop of length 2 (in directed networks, two reverse edges between $i$ and $j$ can be considered as a loop of length 2 as $i \rightarrow j \rightarrow i$ ), the eigenratio will be doubled [50, 51]. Some further works in this direction will be helpful for the in-depth understanding about the role of loops in network synchronization. Moreover, the synchronization processes for networks coupled in different ways are still unclear. As found in the collective phenomena of biological swarms, a very few effective leaders can well organize the whole population [57], implying that better synchronizabilty may be achieved in the same way. Similarly, a recent study shows that partial coupling is more than enough to sustain the coherence of selfpropelled particles [58]. Last but not least, it is recently found that the theory of complementary graphs may also be a key to better understanding and ultimately enhancing the network synchronizability [33]. Therefore, it is believed that how to synchronize a complex network in shorter time with lower cost is a theoretically challenging and practically important research issue, which should be further addressed in the near future.

Acknowledgements G. R. Chen was supported by the Hong Kong Research Grants Council under the CERG Grant CityU No. 1114/05E. M. Zhao, T. Zhou and B. H. Wang were supported by the National Basic Research Project of China (973 Program) under Grant No. 2006CB705500, and by the National Natural Science Foundation of China under Grant Nos. 10635040, 10532060, 10472116 and 70471033.

\section{References}

1. Watts D. J. and Strogatz S. H., Nature, 1998, 393: 440

2. Barabási A. -L. and Albert R., Science, 1999, 286: 509

3. Albert R. and Barabási A. -L., Rev. Mod. Phys., 2002, 74: 47

4. Dorogovtsev S. N. and Mendes J. F. F., Adv. Phys., 2002, 51: 1079

5. Newman M. E. J., SIAM Review, 2003, 45: 167

6. Boccaletti S., Latora V., Moreno Y., Chavez M., and Hwang D. -U., Phys. Rep., 2006, 424: 175

7. Pastor-Satorras R. and Vespignani A., Phys. Rev, Lett., 2001, 86: 3200

8. Yan G., Zhou T., Wang J., Fu Z. -Q., and Wang B. -H., Chin. Phys. Lett., 2005, 22: 510

9. Zhou T., Yan G., and Wang B. -H., Phys. Rev. E, 2005, 71: 046141

10. Motter A. E. and Lai Y. -C., Phys. Rev. E, 2002, 66: 065102

11. Goh K. -I., Lee D. -S., Kahng B., and Kim D., Phys. Rev. Lett., 2003, 91: 148701

12. Zhou T. and Wang. B. -H., Chin. Phys. Lett., 2005, 22: 1072

13. Tadić B., Thurner S., and Rodgers G. J., Phys. Rev. E, 2004, 69: 036102

14. Zhao L., Lai Y. -C., Park K, and Ye N., Phys. Rev. E, 2005, 71: 026125

15. Yan G., Zhou T., Hu B., Fu Z. -Q., and Wang B. -H., Phys. Rev. E, 2006, 73: 046108

16. Lago-Fernández L. F., Huerta R., Corbacho F., and Sigüenza J. A., Phys. Rev. Lett., 2000, 84: 2758

17. Gade P. M. and Hu C. -K., Phys. Rev. E, 2000, 62: 6409

18. Hong H., Choi M. Y., and Kim B. J., Phys. Rev. E, 2002, 65: 026139

19. Barahona M. and Pecora L. M., Phys. Rev. Lett., 2002, 89: 054101 
20. Nishikawa T., Motter A. E., Lai Y. -C., and Hoppensteadt F. C., Phys. Rev. Lett., 2003, 91: 014101

21. Hong H., Kim B. J., Choi M. Y., and Park H., Phys. Rev. E, 2004, 69: 067105

22. Donetti L., Hurtado P. I., and Muñoz M. A., Phys. Rev. Lett., 2005, 95: 188701

23. Zhao M., Zhou T., Wang B. -H., Yan G., Yang H. -J., and Bai W. -J., Physica A, 2006, 371: 773

24. Wang B., Tang H. -W., Zhou T., and Xu Z. -L., arXiv: condmat/0512079

25. Wang X. F. and Chen G., Int. J. Bifurcation Chaos Appl. Sci. Eng., 2002, 12: 187

26. Wang X. F. and Chen G., IEEE Trans. Circuits and Systems I, 2002, 49: 54

27. Pecora L. M. and Carrol T. L., Phys. Rev. Lett., 1998, 80: 2109

28. Barahona M, and Pecora L. M., Phys. Rev. Lett., 2002, 89: 054101

29. Hu G., Yang J., and Liu W., Phys. Rev. E, 1998, 58: 4440

30. Stefański A., Perlikowski P., and Kapitaniak T., Phys. Rev. E, 2007, 75: 016210

31. Duan Z., Chen G., and Huang L., arXiv:0706.2899v1 [cs.NI] 20 Jun. 2007

32. Duan Z., Chen G., and Huang L., arXiv:0706.2990v1 [cs.NI] 20 Jun. 2007

33. Liu C., Duan Z., Chen G., and Huang L., Physica A, accepted, 2007

34. Pecora L. M. and Barahona M., Chaos Complexity Lett., 2005, 1: 61

35. Nishikawa T. and Motter A. E., Phys. Rev. E, 2006, 73: 065106

36. Fan Z. P., Complex Networks: From Topology to Dynamics, PhD Thesis, City University of Hong Kong, 2006

37. Zhao M., Zhou T., Wang B. -H., and Wang W. -X., Phys. Rev. E, 2005, 72: 057102

38. Barthélemy M., Eur. Phys. J. B, 2004, 38: 163
39. Goh K. -I., Kahng B., and Kim D., Phys. Rev. Lett., 2001, 87: 278701

40. Zhou T., Zhao M., and Wang B. -H., Phys. Rev. E, 2006, 73: 037101

41. Bondy J. A. and Murty U. S. R., Graph Theory with Applications, MacMillan Press LTD, London and Basingstoke, 1976

42. Bollobás B., Modern Graph Theory, New York: Springer-Verlag, 1998

43. Xu J. -M., Theory and Application of Graphs, Dordrecht: Kluwer Academic, 2003

44. Newman M. E. J. and Watts D. J., Phys. Rev. E, 1999, 60: 7332

45. Xu J. -M., Topological Structure and Analysis of Interconnection Networks, Dordrecht: Kluwer Academic Publishers, 2001

46. Yin C. -Y., Wang W. -X., Chen G., and Wang B. -H., Phys. Rev. E, 2006, 74: 047102

47. Motter A. E., Zhou C., and Kurths J., Phys. Rev. E, 2005, 71: 016116

48. Motter A. E., Zhou C., and Kurths J., Europhys. Lett., 2005 69: 334

49. Motter A. E., Zhou C., and Kurths J., AIP Conf. Proc., 2005, 776: 201

50. Zhao M., Zhou T., Wang B. -H., Ou Q., and Ren J., Eru. Phys. J. B, 2006, 53: 375

51. Chavez M., Hwang D. -U., Amann A., Hentschel H. G. E., and Boccaletti S., Phys. Rev. Lett., 2005, 94: 218701

52. di Bernardo M., Garofalo F., and Sorrentino F., Int. Bifurca J. \& Chaos, to be published

53. Dorogovtsev S. N., Mendes J. F. F., and Samukhin A. N., Phys. Rev. Lett., 2000, 85: 4633

54. Krapivsky P. L. and Redner S., Phys. Rev. E, 2001, 63: 066123

55. Newman M. E. J., Phys. Rev. Lett., 2002, 89: 208701

56. Newman M. E. J., Phys. Rev. E, 2003, 67: 026126

57. Couzin L. D., Krause J., Franks N. R., and Levin S. A. Nature, 2005, 433: 513

58. Zhang H. -T., Chen M., and Zhou T., arXiv:0707.3402 